\title{
Effects of multi-wall carbon nanotubes on structural and mechanical properties of poly(3-hydroxybutyrate)/chitosan electrospun scaffolds for cartilage tissue engineering
}

\author{
SAEED KARBASI* and ZAHRA MOHAMMAD ALIZADEH \\ Department of Biomaterials and Tissue Engineering, School of Advance Technology in Medicine, Isfahan University of \\ Medical Sciences, Isfahan 81746 73461, Iran \\ *Author for correspondence (Karbasi@med.mui.ac.ir)
}

MS received 17 August 2016; accepted 20 February 2017; published online 22 September 2017

\begin{abstract}
Poly(3-hydroxybutyrate) (PHB)/chitosan electrospun scaffold was recently prepared for cartilage tissue engineering purpose. The drawback of this scaffold was its low mechanical properties. This study was carried out to see if addition of multi-wall carbon nanotubes (MWNTs) to PHB/chitosan polymeric blend can show better mechanical and structural properties. To do this, three different amounts of MWNTs $(0.5,0.75$ and $1 \mathrm{wt} \%)$ were added to PHB/chitosan solution. Then, the prepared solution was electrospun. The fibre's diameter and uniformity were assessed by SEM. The solution components entity authenticity was approved by FTIR. The porosity assessment was illustrated by a porous structure with $81-83 \%$ porosity. Water contact angle (WCA) test showed the decrease in contact angle with the increase in MWNTs. Mechanical property results showed the strength of about 4-10 MPa for composites with different percentages of MWNTs, while $\mathrm{PHB} / \mathrm{chitosan}$ showed the strength of $3 \mathrm{MPa}$. Actually, the mechanical properties of composite showed higher values when compared to polymeric blend scaffold. All the results reveal that the addition of $1 \mathrm{wt} \%$ of MWNTs to the polymeric solution is the most optimal percentage whose values are close to cartilage properties.
\end{abstract}

Keywords. Poly(3-hydroxybutyrate); multi-wall carbon nanotubes; chitosan; tissue engineering; electrospinning.

\section{Introduction}

Cartilage is the most affordable replacement for skeletal tissues. In human body, this tissue is placed either in loading or non-loading areas. It is composed of cells, fibres and amorphous matrix. Chondrocytes are the most important cellular source in cartilage joints $[1,2]$. Because of the lack of blood source and undifferentiated cells, and also the slow rate of the proliferation of chondrocytes, cartilage regeneration is restricted [3]. Tissue engineering is a new developing technique which can help to a promising cartilage regeneration in in vitro condition. Cartilage tissue engineering needs not only the living cells, but also the interaction among chondrocytes, extracellular scaffold and bioenvironmental factors. For in vitro tissue engineering, the cultured cells are grown on biodegradable and bioactive biomaterials called scaffolds. Since the material surface properties effect directly on cellular behaviour, the scaffold structure has a key role in tissue formation process. The scaffolds provide not only a basic structure for cell growth and tissue formation, but also to determine how to transport the materials [4]. Synthetic and natural biomaterials which are used for scaffold fabrication, is chosen based on their biocompatibility, biodegradability and mechanical properties [5]. Generally, among the different kinds of material scaffolds, polymeric scaffolds showed the best biocompatibility and cell viability properties similar to human body structural parts, although they have shown weak mechanical characteristics due to the nature of polymers. These properties go weaker by the creation of porosity, which is needed for the cell's three-dimensional growth. This point made the researchers capable of fabricating composites from organic and inorganic materials [6]. PHB-chitosan polymeric blend scaffold was studied recently by Sadeghi et al [7] and the results showed good biocompatibility and cell behaviour, although the mechanical properties and hydrophilicity are not significant. One of the methods to improve these properties is making composite scaffold by the addition of nanoparticles and nanostructures to it, so that it does not affect negatively on porosity. To this end, it was suggested to add a ceramic component which can enhance the mechanical properties and causes the water contact angle (WCA) to decline. Carbon nanotubes (CNT) have one of the simplest chemical composition and atomic bonding configuration and also have more variations among nanostructure materials [8]. The mixture of size, structure and topology gives carbon nanotubes significant mechanical properties such as high stability, strength and toughness, also surface properties and elastic flexibility [9]. Since CNTs are toxic materials when exposed to cells, functionalization is a promising way to solve these kinds of problems perfectly. Also, the presence of functional groups leads to decrease in the van der Waals interactions between the CNTs, which cause the tubes to meet each other 

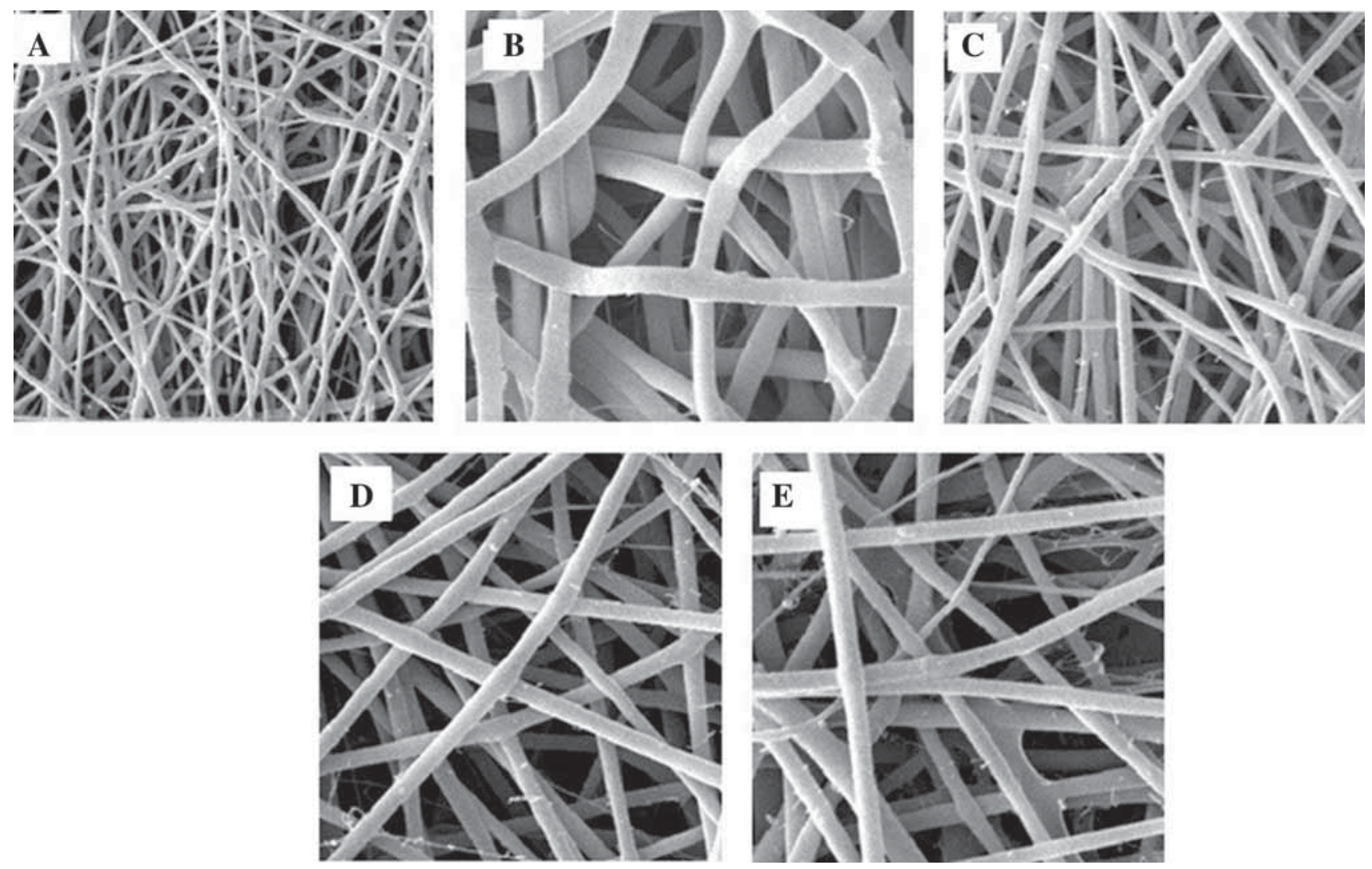

Figure 1. SEM was undertaken for five samples which were consisted of two control samples and three nanocomposite samples with different MWNTs percentages. These samples were respectively: (a) pure PHB, (b) PHB-chitosan blend composite, (c) PHBchitosan/0.5\% MWNTs, (d) PHB-chitosan/0.75\% MWNTs and (e) PHB-chitosan/1\% MWNTs.

separately [10]. Generally, the addition of CNTs has more effect on mechanical properties when compared to other nanoparticles when used in lower percentages [11]. Kharaziha et al [12] have studied on the electrospun gelatin-CNT nanofibre scaffold which showed significant mechanical properties and cardiac cell viability. In another study, Shi et al [13] have illustrated that electrospun polypropylene fumaratesingle wall carbon nanotube (SWNT) scaffold has increased biocompatibility and mechanical properties. According to these studies, one of the effective methods to have uniform fibres with up to $80 \%$ porosity, is electrospinning which is based on the fibre spinning by exerting a high voltage electric field to the polymeric solution [14].

According to these details, this study was carried out to evaluate the mechanical and physical properties which were acquired by the addition of MWNTs to PHB-chitosan polymeric blend scaffold to use it in cartilage tissue engineering.

\section{Materials and methods}

\subsection{Materials}

PHB (Sigma, Aldrich, USA), medium molecular weight chitosan with deacetylation degree of $75-85 \%$ (Sigma, Aldrich,
USA), MWNTs (US Research Nanomaterials Incorporation, USA), trifluoroacetic acid (TFA) (Merck, Germany) were employed in this study.

\subsection{Preparation of electrospun scaffold}

As per Sadeghi et al's [7] study, initially, a $9 \mathrm{wt} \%$ PHBchitosan solution was made in TFA solvent. Then, three different wt $\%(0.5,0.75$ and 1$)$ of functionalized carbon nanotubes were added to a little amount of TFA solvent. To disperse nanoparticles and to avoid accumulation, the mentioned solution was sonicated by Sonicator (SYCLON, SKL-950W) for $12 \mathrm{~s}$. This solution was added to PHB-chitosan solution and was allowed for about $15 \mathrm{~min}$ to dissolve. Finally, the acquired solution was taken into a $1 \mathrm{cc}$ insulin syringe and it was set in its place. The distance, flow-rate and voltage were just optimized. According to optimization, the distance between the syringe and the collector was adjusted to $15 \mathrm{~cm}$, the flow rate was set at $0.5 \mathrm{mlh}^{-1}$ and the voltage of $15.0 \mathrm{kV}$ was exerted on it which could produce a uniform jet exuded from needle and a random fibre structure was formed on the collector. One-way ANOVA analysis was used to compare the samples. 


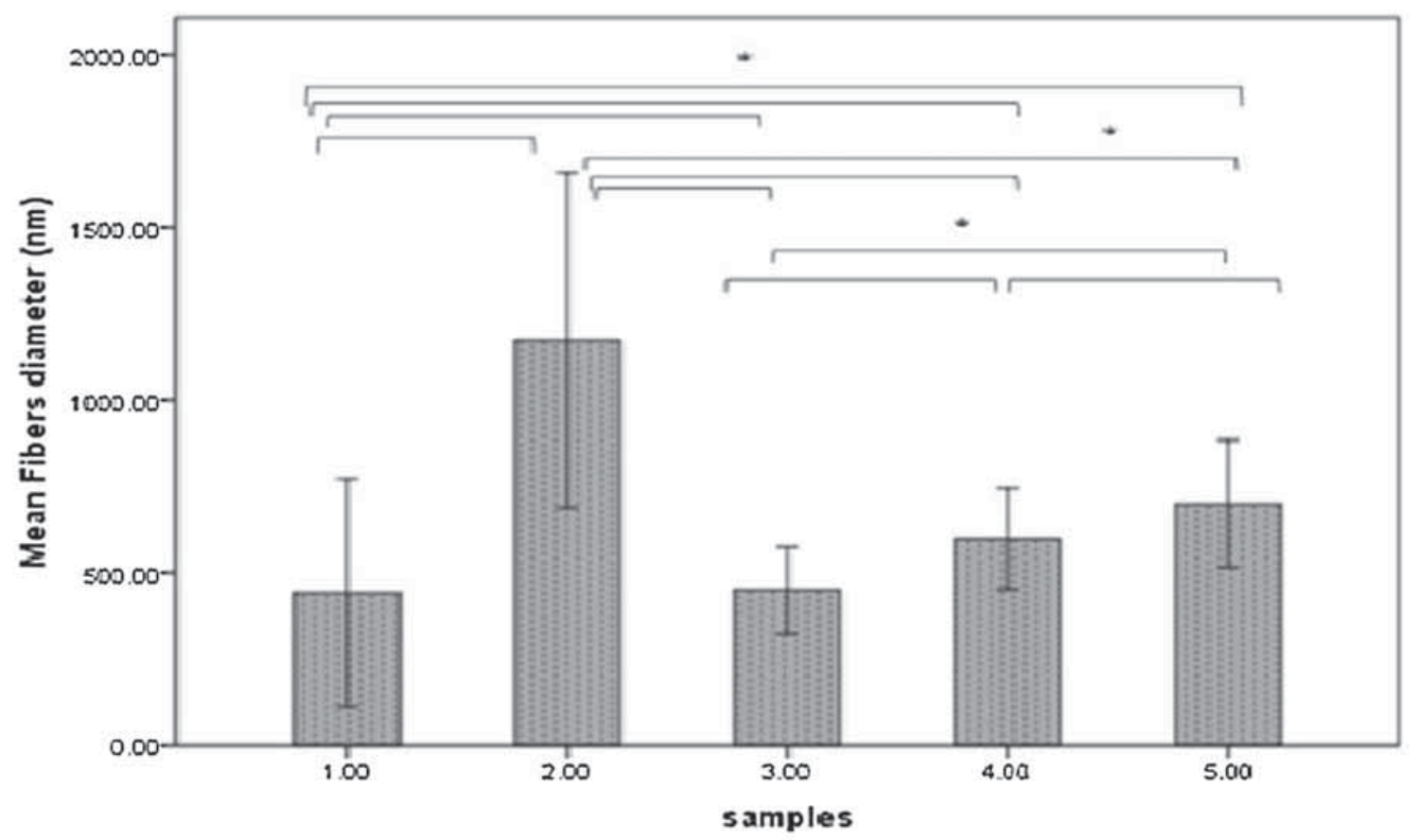

1)PHB, 2)PHB-Chitosan, 3)PHB-Chitosan/0.5\% MWNTs, 4)PHB-Chitosan/0.75\% MWNTs, 5)PHBChitosan $/ 1 \%$ MWNTs

Error bars: $95 \% \mathrm{Cl}$

Figure 2. Fibres diameter distribution histogram. Fibres diameter was significantly $(P<0.05)$ lower in scaffolds with MWNTs content of 0.5 and 0.75 and $1 \mathrm{wt} \%$ in comparison with PHB-chitosan. Also, the diameter of the fibres of $1 \mathrm{wt} \%$ MWNTs was meaningfully more than 0.5 and $0.75 \mathrm{wt} \%(* P<0.001)$.

Table 1. Illustration of porosity results in four layers of each nanocomposite scaffold.

\begin{tabular}{lccccc}
\hline $\begin{array}{l}\text { Scaffold } \\
\text { porosity }\end{array}$ & Pure PHB & PHB-chitosan & $\begin{array}{l}\text { PHB-chitosan/ } \\
(0.5 \%) \text { MWNTs }\end{array}$ & $\begin{array}{l}\text { PHB-chitosan/ } \\
(0.75 \%) \text { MWNTs }\end{array}$ & $\begin{array}{l}\text { PHB-chitosan/ } \\
(1 \%) \text { MWNTs }\end{array}$ \\
\hline \% First layer & 83.70 & 85.57 & 83.70 & 82.92 & 81.70 \\
\% Second layer & 45.73 & 44.90 & 45.73 & 48.07 & 49.46 \\
\% Third layer & 21.79 & 20.83 & 21.79 & 24.32 & 0.001 \\
\hline
\end{tabular}

\subsection{Evaluation of scaffold morphology}

The evaluation of scaffold fibres morphology and structure was accomplished by scanning electron microscopy (SEM, AIS-2100). The Image $\mathbf{J}$ software was used for fibre diameter measurement. The porosity of scaffolds was evaluated by MATLAB software program [7]. These two approaches were accomplished on SEM photomicrographs. Fourier transform spectrophotometry (FTIR) (Bruker alpha, Germany) evaluation was also undertaken to prove the existence of materials which were used in electrospinning.

\subsection{WCA measurement}

The hydrophilicity of electrospun scaffolds was examined by WCA assay. According to this assay, a droplet of double deionized water was dropped on scaffold surface and then the picture was taken by the camera (KSV Cam 200 Instrument, Finland). Then, the picture was evaluated by Image J software to see the angle between the water drop and scaffold.

\subsection{Mechanical properties measurement}

The mechanical test was carried out according to standard ASTM-F1634 and ASTM-D882. The scaffold was cut into $10 \times 30 \mathrm{~mm}^{2}$ pieces, and each of them was placed in the frame which was made earlier. The frame was embedded in the mechanical test device (INSTRON-5566, USA). The tensile rate of the device was $5 \mathrm{~mm} \mathrm{~min}^{-1}$ and load cell was $50 \mathrm{~N}$. 


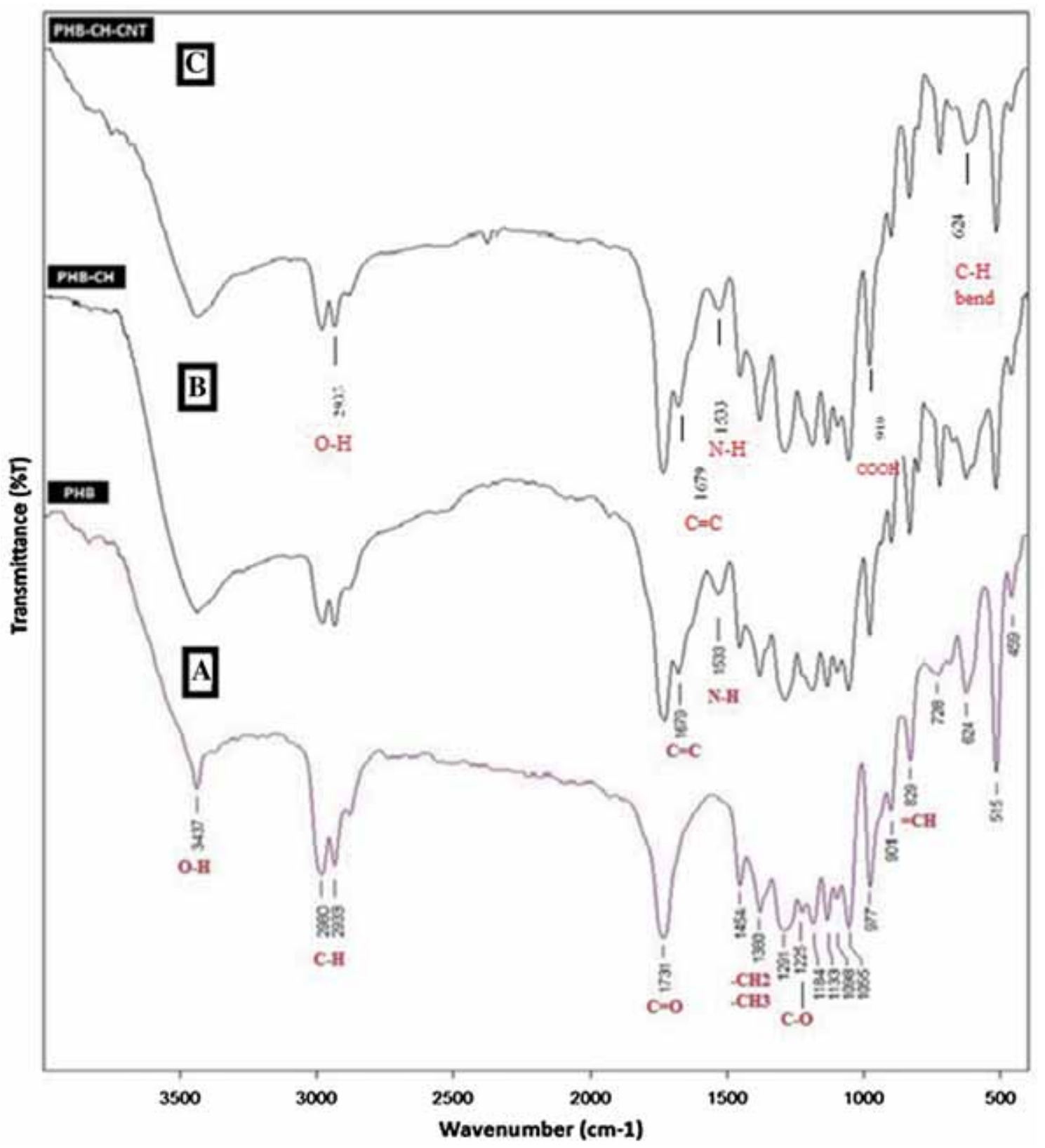

Figure 3. A comparison between FTIR of PHB, PHB-chitosan and PHB-chitosan/1\% MWNTs.

\section{Results and discussion}

\subsection{Structural properties}

3.1a SEM: The SEM photomicrographs have shown uniform fibre structures without any bead (figure 1). The results illustrated that an increase in MWNTs content can lead to an enhancement of fibre diameter, even though, the addition of chitosan can cause a larger fibre diameter than pure PHB. Also, the sonication of MWNTs solution helped the carbon nanotubes to disperse perfectly into the polymer chains [15], so that it was electrospun as well. Scott et al [16] evaluated the effect of solution concentration on electrospun fibres diameter. The results showed that as polymeric solution concentration increases, fibres diameter increases gradually. In this study, with the addition of MWNTs to polymeric solution, the concentration decreases, so the fibres diameter reduces subsequently. Consequently, all the PHB-chitosan/MWNTs nanocomposites revealed homogenous structure with uniform fibres. Fibres diameter was significiantly $(P<0.001)$ lower in scaffolds with MWNTs content of $0.5,0.75$ and $1 \mathrm{wt} \%$ in comparision with $\mathrm{PHB}-$ chitosan. Also, the fibres diameter of $1 \mathrm{wt} \%$ MWNTs was significantly $(P<0.001)$ more than 0.5 and $0.75 \mathrm{wt} \%$. PHB-chitosan $/ 0.5 \%$ MWNTs has produced lower thickness in comparison with the other two percentages. These fibres diameter was about 460-500 nm, while the 

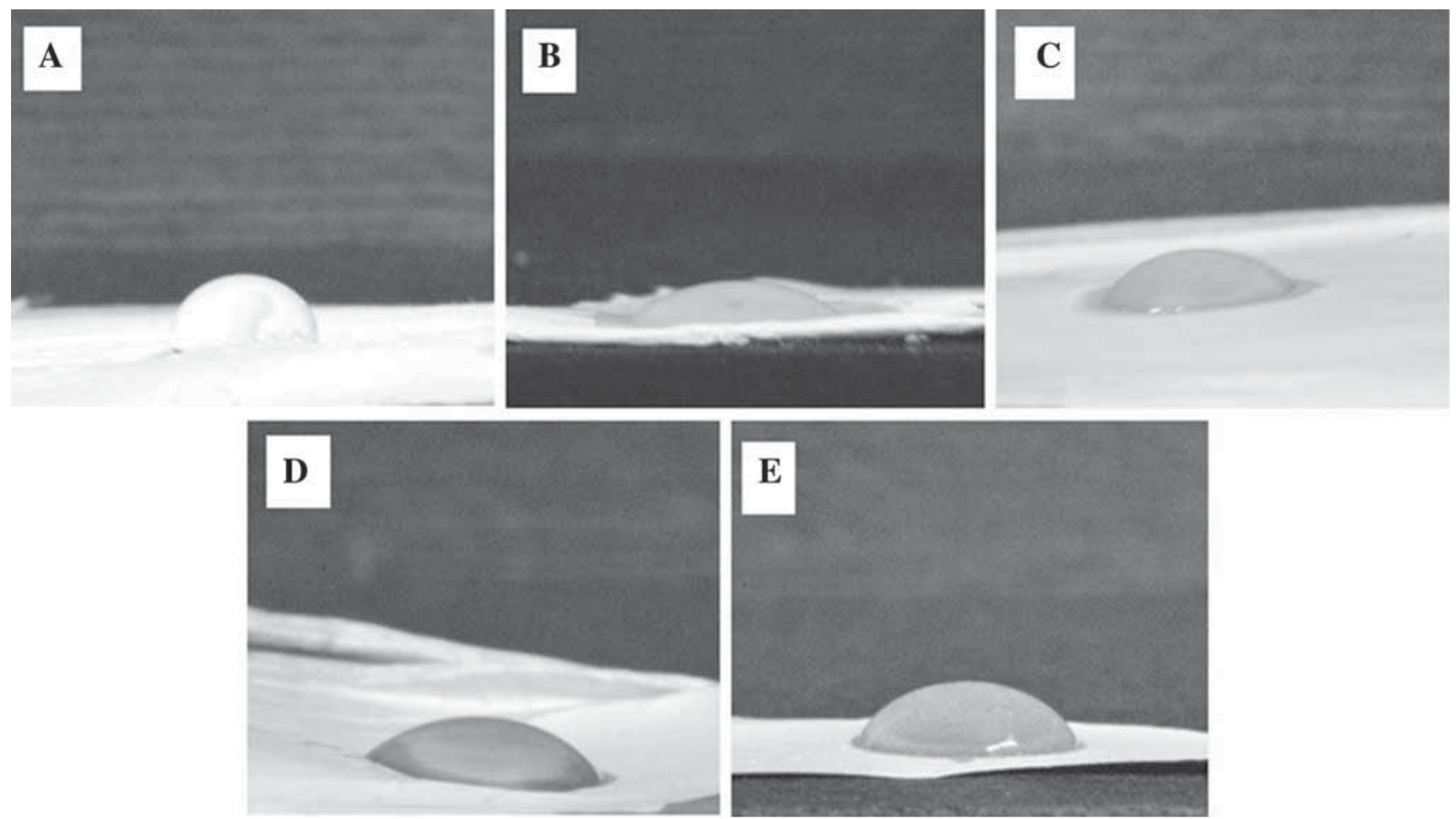

Figure 4. WCA assay was undertaken by professional camera for (a) pure PHB, (b) PHB-chitosan, (c) PHB-chitosan/0.5\% MWNTs, (d) PHB-chitosan/0.75\% MWNTs and (e) PHB-chitosan/1\% MWNTs nanocomposite scaffolds.

Table 2. WCA standard deviation for different amounts of MWNTs.

\begin{tabular}{lcc}
\hline$P$-value & WCA $\left(^{\circ}\right)$ & Scaffold sample \\
\hline 0.001 & $79.28 \pm 2.12$ & PHB \\
& $45.31 \pm 2.57$ & PHB-chitosan \\
& $32.82 \pm 4.10$ & PHB-chitosan/0.5\% MWNTs \\
& $35.46 \pm 0.95$ & PHB-chitosan/0.75\% MWNTs \\
& $42.26 \pm 1.78$ & PHB-chitosan/1\% MWNTs \\
\hline
\end{tabular}

0.75 and $1 \%$ MWNTs respectively, showed about 540 and $700 \mathrm{~nm}$ (figure 2).

3.1b Porosity: Table 1 shows the samples porosity. According to this table, among all the samples, PHB-chitosan has had more porosity than others, although, all the scaffolds had the porosity over $80 \%$ which is a promising outcome for tissue engineering purposes [17]. This can be the result of its fibres' thickness. Usually, as the fibres go thicker, the porosity gets larger and its percentage goes up [18]. While a comparison between the nanocomposites with different amount of MWNTs, has shown an increase in porosity by decreasing in the MWNTs content.

While a comparison between the nanocomposites with different amounts of MWNTs has shown an increase in porosity by decreasing the MWNTs content. According to this fact, the porosity results in the first layer were significant $(P<0.001)$. In the next layers, there are porosities about $20-40 \%$. The presence of pores in percentages up to $20 \%$ in the other three layers suggest that there are interconnective pores which are suitable for cells to permeate (table 1).

3.1c FTIR: FTIR was undertaken to illustrate the constituents of electrospun scaffolds (figure 3). A spectrum was seen in $3436 \mathrm{~cm}^{-1}$, which is related to the existence of MWNTs in PHB-chitosan polymeric blend scaffold and in $2900 \mathrm{~cm}^{-1}$ which is related to $-\mathrm{COOH}$ in MWNTs functional group (figure $3 \mathrm{c}$ ). There are peaks seen in 1731, 728, 1225 and $1380 \mathrm{~cm}^{-1}$, which are correlated to $-\mathrm{COO}-, \mathrm{C}-\mathrm{H}, \mathrm{C}-\mathrm{O}$, $-\mathrm{CH}_{2} \mathrm{CH}_{3}$ and $\mathrm{CH}_{3}$ bonds (figure $3 \mathrm{a}$ ). These peaks are seen in all the three spectrums and indicate the PHB presence in all the three kinds of scaffolds. The two spectra of 1679 and $1533 \mathrm{~cm}^{-1}$ were observed in chitosan structure of figure $3 \mathrm{~b}$, which is related to $\mathrm{C}=\mathrm{C}$ and $-\mathrm{CONH}-$ bonds, respectively. The FTIR result has proved the absence of TFA solvent in the nanocomposite scaffolds, which was evaporated during the electrospinning process.

3.1d Contact angle: WCA was performed for five samples which are shown in figure 4. PHB is a hydrophobic polymer as it was proved earlier [19]. In this study, PHB was evaluated and the results showed an average degree of 79.28. Since the chitosan is a natural polymer, it is a hydrophilic one [20]. Hence, it is expected that by the addition of chitosan to PHB, 


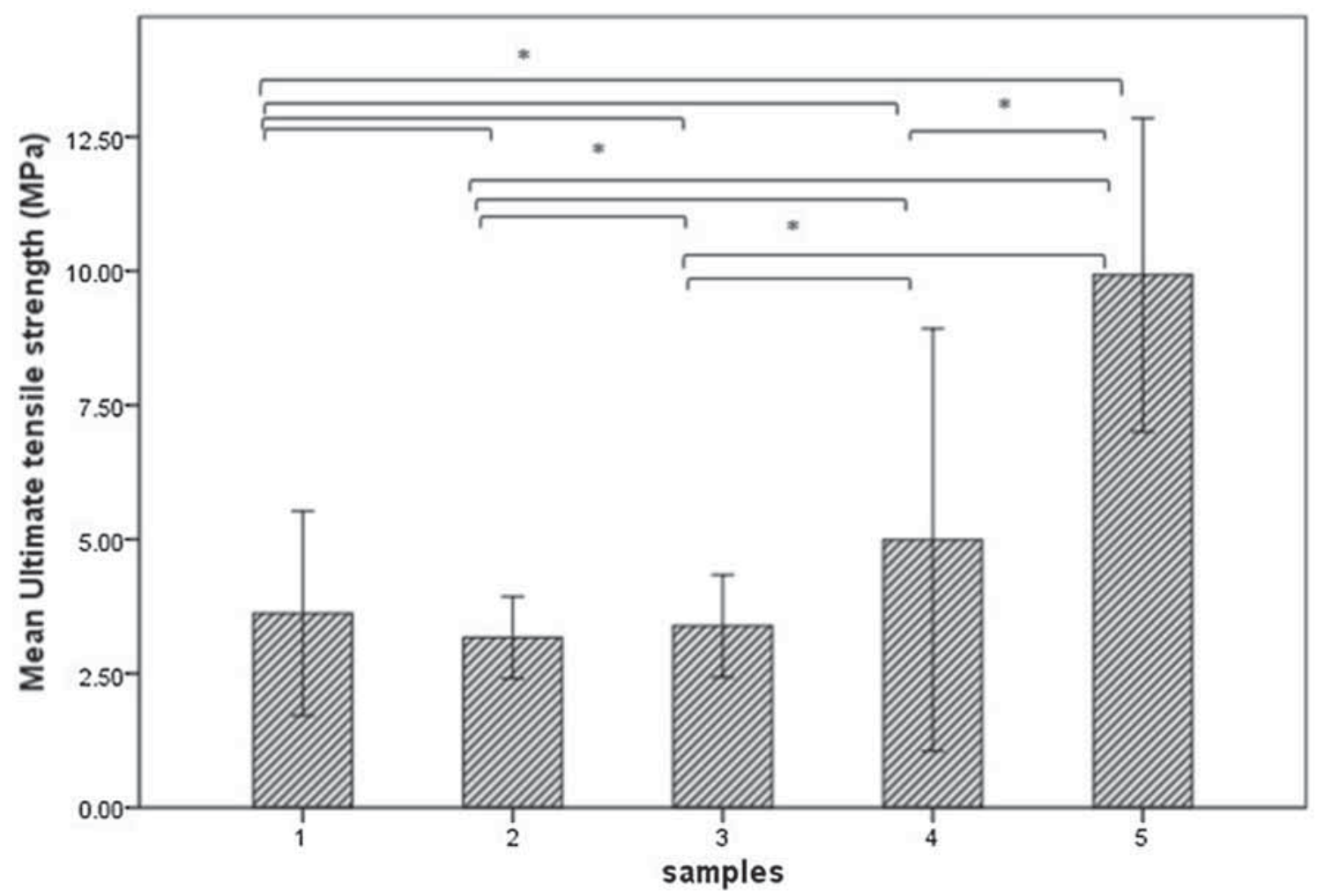

1)PHB, 2)PHB-Chitosan, 3)PHB-Chitosan/0.5\% MWNTs, 4)PHB-Chitosan/0.75\% MWNTs, 5)PHBChitosan $/ 1 \%$ MWNTs

\section{Error bars: $95 \% \mathrm{Cl}$}

Figure 5. Illustration of change of ultimate tensile stress by changes in MWNTs contents in two repetitions. The tensile strength was significantly $(P<0.001)$ high for $1 \mathrm{wt} \%$ MWNTs.

WCA gets lower, whose results showed an average degree of 45.31, which proved this fact [7]. On the other hand, carbon nanotubes are intrinsically hydrophobic, but when they functionalize, their functional group can make them to show hydrophilic properties [21]. A comparison between different amounts of MWNTs is illustrated in table 2. WCA for $1 \%$ MWNTs was 42.26, which is more than two others. This angle for 0.5 and $0.75 \%$ MWNTs are respectively, about 32.82 and 35. Actually, more MWNTs contents bring more WCA. The reason for high WCA of $1 \%$ MWNTs rather than two others can be the hydrophobic nature of MWNTs, which overcome the hydrophilic nature of chitosan. In low percentages, the effect of chitosan makes scaffold to have WCA lower than PHB-chitosan (figure 4).

\subsection{Mechanical properties}

As the results of the mechanical test, which are shown in figure 5, the ultimate tensile strength of nanocomposite increased by the addition of the MWNTs content in higher percentages. Hence, the PHB-chitosan/1\% MWNTs scaffold had the highest ultimate tensile strength, about $10 \mathrm{MPa}$, among the others. This value is about two times more than $0.75 \%$
MWNTs, and 2.5 times more than $0.5 \%$. These consequences are shown the effective role of chitosan in decreasing the mechanical properties, which needs more MWNTs entity to improve. Also, the results of the pure PHB and PHB-chitosan scaffolds are compared [8].

In this assay, it was illustrated that by the addition of chitosan to PHB solution, the scaffold was lost its mechanical protection. Mattioli et al [22] studied on PCL-CNT composites, which showed that by an increase in CNT contents, elastic modulus initially increases, but then, it decreases subsequently. Liu et al [23] studied chitosan/CNT nanocomposites in three percentages $(0.5,1$ and $1.5 \%)$ and the results illustrated an enhancement in tensile strength and elastic modulus by the addition of functionalized CNT contents, which are significant $(P<0.001)$. Also, Guo et al [24] evaluated mechanical properties of PHBV/MWNTs composites. They revealed that by an increase in MWNTs content, the tensile strength increases correspondingly first. In $1 \mathrm{wt} \%$ of MWNTs, there was a peak in tensile strength and then it was decreased. On the other hand, elastic modulus of healthy cartilage in regions with meniscus cover is in the range from $2.13 \pm 0.74$ up to $5.13 \pm 1.91 \mathrm{MPa}$ [25]. As a result of this assay, PHB-chitosan/1\% MWNTs nanocomposite scaffold 
showed the most favourable mechanical properties among others. The elastic moduli values for this scaffold are in the range of $1.13 \pm 0.32 \mathrm{MPa}$, which are close to cartilage values. These results showed that the addition of CNT content to polymeric blend solution leads to an increase in the values of elastic moduli.

\section{Conclusions}

MWNTs were added to PHB-chitosan solution and electrospun. Fibres diameter was thinner than earlier and they produced optimum porosity for scaffold. The hydrophilicity reduced in the presence of MWNTs. Mechanical properties are almost close to cartilage characteristics. Consequently, PHB-chitosan/MWNTs scaffold showed suitable structural and mechanical behaviours which can be used for cartilage tissue engineering.

\section{References}

[1] Kaufman M R and Tobias G W 2003 Clin. Plast. Surg. 30539

[2] Freeman M A R 2011 Adult articulate cartilage

[3] Mort J S and Billington C J 2001 Arthritis Res. 3337

[4] Hutmacher D 2001 J. Biomater. Sci. Polym. Ed. 2107

[5] Woodfield T B, Malda J, Wijn J, Peters F, Riesle J and Blitterswijk C A 2004 Biomaterials 254149

[6] Hollister S J 2005 Nat. Mater. 4518

[7] Sadeghi D, Karbasi S, Razavi S, Mohammadi S, Shokrgozar M A and Bonakdar S 2016 J. Appl. Polym. Sci. 13344171
[8] Dai H 2002 Acc. Chem. Res. 351035

[9] Wildgoose G G, Banks E C, Leventis C H and Compton G R 2005 Microchim. Acta 152187

[10] Balasubramanian K and Burghard M 2005 Small 1180

[11] Harrison B S and Atala A 2007 Biomaterials 28344

[12] Kharaziha M, Shin S R, Nikkhah M, Topkaya S N, Masoumi N, Annabi N et al 2014 Biomaterials 357346

[13] Shi X F, Hudson J L, Spicer P P, Tour J M, Krishnamoorti R and Mikos A G 2006 Biomacromolecules 72237

[14] Fujihara K, Kotaki M and Ramakrishna S 2005 Biomaterials 26 4139

[15] Wang S-F, Shen L, Zhang W-D and Tong Y-J 2005 Biomacromolecules 63067

[16] Sell S, Barnes C, Simpson D and Bowlin G 2008 J. Biomed. Mater. Res. Part A 85115

[17] Mohammadian M and Haughi A K 2014 Bulg. Chem. Commun. 46545

[18] Boland E D, Coleman B D, Barnes C P, Simpson D G, Wnek G E and Bowlin G L 2005 Acta Biomater. 1115

[19] Chen G-Q and Wu Q T 2005 Biomaterials 266565

[20] Calvo P and Remunan-Lopez C 1997 J. Appl. Polym. Sci. 63 125

[21] Park J M, Wang Z J, Jang J H, Gnidakoung J R N, Lee W I, Park J K et al 2009 Composites. Part A: Appl. Sci. Manuf. 40 1722

[22] Mattioli-Belmonte M, Vozzi G, Whulanza Y, Seggiani M, Fantauzzi V, Orsini G et al 2012 Mater. Sci. Eng. C 32152

[23] Liu Y L, Chen W H and Chang Y H 2009 Carbohyd. Polym. 76 232

[24] Guo C, Zhou L and Lv J 2013 Polym. Polym. Compos. 21449

[25] Thambyah A, Nather A and Goh J 2006 Osteoarthr. Cartilage 14580 